\title{
Modeling and simulation of air-gapped current transformer based on Preisach Theory
}

\author{
Ya Hui Wu*, Xin Zhou Dong and Sohrab Mirsaeidi
}

\begin{abstract}
Current Transformer (CT) modeling, by which CT's characteristics can be studied has a significant importance in CT selection and design. In spite of numerous studies about closed-core CT model, only a few works have been conducted on air-gapped ones with the following problems: models of which required data is easily-accessible, have poor simulations of magnetization process; on the contrary, models which have satisfactory simulations, are hard to be established because of the hard-to-get required data. Therefore, based on Preisach Theory, a novel air-gapped CT model is deduced from the closed core $C T$ model. The proposed model is accurate and can be established easily. The saturation and remanence properties of closed-core CT and air-gapped CT are simulated and compared.
\end{abstract}

Keywords: CT modeling, Air gap, Preisach Theory, Saturation, Remanence

\section{Introduction}

Current transformer is a bridge between the primary and secondary equipment in power system, utilized for transferring large currents proportionally to currents with small amplitudes for secondary measuring and monitoring equipment and relay protective equipment. In case of a short-circuit incident, as the power system capacity and the voltage level increase, the short-circuit current will reach tens of times or even hundreds of times of CT rated current, leading to CT saturation. In that situation, CT cannot transfer the primary current correctly, which causes misoperation or mal-operation of protection equipment and greater errors of fault location equipment. In response to this, manufacturers open a small air gap on the core of CT, which is about one-thousandth of the total length of the magnetic circuit. Among the CTs that have been widely put into use, the PR-type and the TPY-level of the TP-type are those who have air-gaps in their cores. The air-gapped CT can guarantee that the errors are within the permissible range of the relay protection in the steady state and

\footnotetext{
* Correspondence: wuyh04@gmail.com

State Key Laboratory of Control and Simulation of Power Systems and Generation Equipment, Department of Electrical Engineering, Tsinghua University, Beijing 100084, China
}

the transient state. Establishing the simulation model of air-gapped CT and studying its transient characteristics will help to understand the role of air gap more accurately, and to guide the selection and design of air-gapped CT.

Currently, there are large number of papers on the closed-core CT modeling. The advantages and disadvantages of different models and their application ranges are introduced in detail in [1]. However, there are only a few studies have been accomplished on modeling air-gapped $\mathrm{CT}$. In [2], the real-time magnetization curve is generated by hysteresis loop compressing method, and the real-time excitation inductance is calculated by using the arc tangent function to fit the limiting hysteresis loop, in which way the air-gapped CT model is established on the PSCAD/EMTDC platform. This model can simulate the saturation characteristics of air-gapped CT, but the simulation of the core is not accurate enough. In [3], the air-gapped CT model is established based on Jiles-Atherton (JA) Theory. This model can accurately simulate the effect of air gap on the magnetization process. However, the parameters such as regional coupling coefficient, thermal coefficient, regional flexibility, direction coefficient, which are required for the JA theoretical model, are often hard 
to accurately obtained and can only be approximated. Theoretical model of air-gapped CT given in [4] is established by deducing the relationship between the equivalent excitation inductance of an air-gapped CT and that of a closed-core CT, using principles of circuits and magnetic circuits. The model can be used to deduce the performance of air-gapped CT, to demonstrate the role of air gap, but cannot give the specific value of the excitation inductance; hence, it cannot be used for simulation. According to [4], using the finite element analysis software ANSYS to calculate the real-time excitation inductance, CT equivalent equation is solved for numerical solution [5], but the process is complex, and it is not conducive to simulation either.

Preisach Theory, which is a phenomenological approach to explain the magnetization process [6], can fit very well with the experimental data. Numerous papers and data have testified the feasibility and accuracy of Preisach Theory [7-11]. A TPY-level model is proposed in [10], which is complicated and needs lots of experimental data due to the involving with identification and calculation of Everett function. Based on Preisach magnetization Theory, an air-gapped CT model is proposed in this paper, which is deduced from the closed-core CT model. The model can correctly simulate the magnetization process of air-gapped core, and the data needed for modeling are easy to be obtained. Model parameters can be set arbitrarily, and simulation can be performed easily. The saturation and remanence characteristics of the air-gapped CT model and the closed-core CT model of the same structure and parameters are compared in simulation.

\section{Methods}

\subsection{Introduction to Preisach magnetization theory}

Preisach magnetization Theory is proposed by the German physicist F. Preisach in 1935. After continuous improving and perfecting, it has gradually formed a guiding theory to the ferromagnetic material modeling $[8,12-15]$.

Preisach Theory holds that the ferromagnetic material consists of numerous magnetic dipoles, each of which has its own saturation characteristics, as shown in Fig. 1. Macroscopic magnetization shown by ferromagnetic material is the accumulation of the microscopic magnetization of all the magnetic dipoles that make up it. Each magnetic dipole has its own positive saturation threshold $\alpha$ and negative saturation threshold $\beta$. As the name implies, when the applied magnetic field strength is greater than the positive saturation threshold $\alpha$, the magnetic dipole enters the positive saturation state. When the

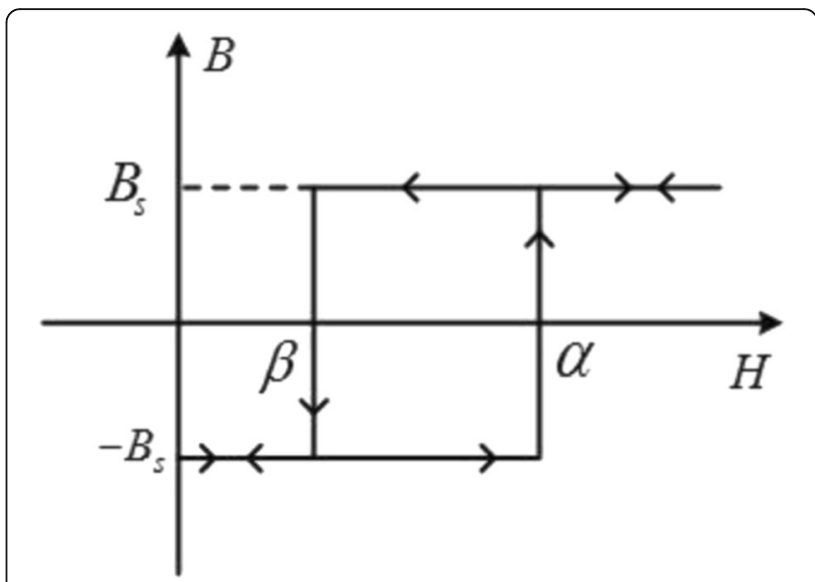

Fig. 1 Magnetization characteristics of the magnetic dipole

applied magnetic field strength is less than $\beta$., the magnetic dipole enters the negative saturation state, and when the applied magnetic field strength is between $\beta$ and $\alpha$, both state may magnetic dipole be in, depending on the historical change of the magnetic field, which manifests itself as a memory characteristic of ferromagnetic matter.

While using Preisach Theory to calculate the core magnetic flux density, it is only necessary to calculate the difference between the integral of the magnetic dipole distribution density in region $S_{+}$and the integral in region $S_{-}$. in the triangle ABC in Preisach diagram as Fig. 2 shows.

$$
B(H)=B_{S}\left(\iint_{S_{+}} \mu(\alpha, \beta) d \alpha d \beta-\iint_{S_{-}} \mu(\alpha, \beta) d \alpha d \beta\right)
$$

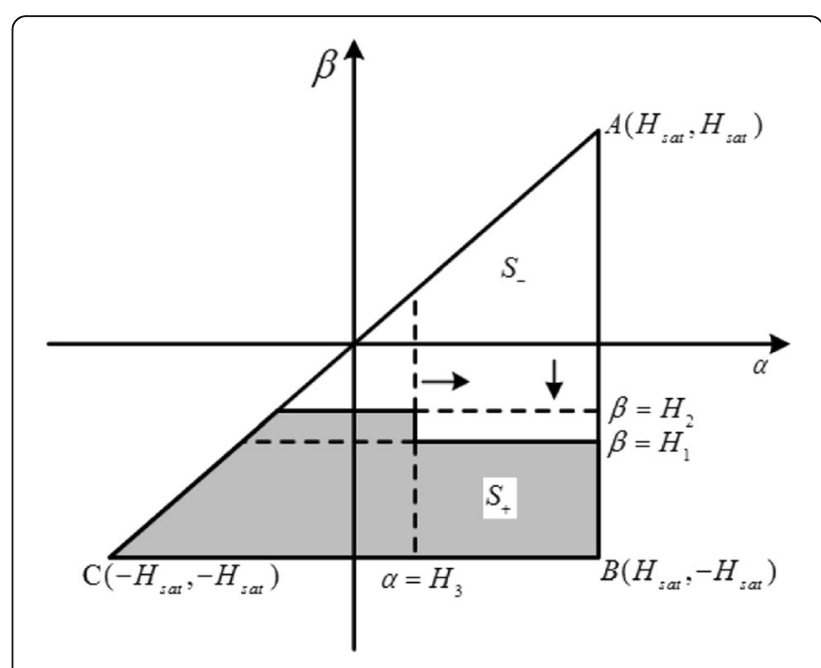

Fig. 2 A typical Preisach diagram 
In Equation (1), $\mu(\alpha, \beta)$ represents the distribution density of magnetic dipoles, and should have the following properties:

1) $\alpha<\beta, \mu(\alpha, \beta)=0$;

2) if $\alpha>H_{\text {sat }}$ or $\beta<-H_{\text {sat }}, \mu(\alpha, \beta)=0$

Where, $H_{\text {sat }}$ is positive saturation field strength;

3) $\mu(\alpha, \beta)=\mu(-\beta,-\alpha)$

The changes of magnetic field strength $H$ influence the value of flux density $B$ in the following way: when $H$ is greater than $H_{\text {sat }} S_{+}$covers the entire triangle area, at which time the core is in a positive saturation state. When $H$ is smaller than $-H_{s a b} S_{-}$, covers the entire triangle area, at which time the core is in a negative saturation state. When $H$ decreases, triangle $\mathrm{ABC}$ will be swept down by a straight line perpendicular to the $\beta$-axis, $\beta=H$, then all the magnetic dipoles whose $\beta$ value is greater than $H$ will enter the negative saturation state, so the swept area is covered by $S_{-}$. When $H$ increases, triangle ABC will be swept down by a straight line perpendicular to the $\alpha$ axis, $\alpha=H$, then all the magnetic dipoles whose $\alpha$ value is smaller than $H$ will enter the positive saturation state, so the swept area is covered by $S_{+}$.

Since it is hard to determine $\mu(\alpha, \beta)$ and to calculate double integral, reference [8] put forward the assumption that variables in $\mu(\alpha, \beta)$ can be separated.

$$
\mu(\alpha, \beta)=\mu_{\alpha}(\alpha) \mu_{\beta}(\beta)
$$

So double integral is transferred into single integral, and formulas (3, 4 and 5), can be deduced according to the shapes of different Preisach diagrams [16].

$$
\begin{aligned}
& B_{i}(H)=\left(B_{d}(H) / 2+B_{u}(H) / 2\right)^{2}\left(B_{d}(H)\right)^{-1} H \geq 0 \\
& \sqrt{B_{d}(H)}=F(-H) \\
& B_{u}(H)=B_{d}(H)-2 F(H) F(-H)
\end{aligned}
$$

Where, $B_{d}(H), B_{u}(H), B_{i}(H)$ is the limiting descending branch, the limiting ascending branch and the virgin curve respectively. According to the symmetric properties of the limiting hysteresis loop:

$$
B_{u}(H)=-B_{d}(-H)
$$

Assuming that $\left(H_{1}, B_{1}\right)$ is the local extremum of magnetization trajectory, the descending branch and ascending branch from it can be calculated by:

$$
\begin{aligned}
& B_{H_{1}, B_{1}}{ }^{u}=-B_{d}(-H)-B_{d}\left(H_{1}\right)+B_{1}+2 \mathrm{~F}\left(-H_{1}\right) \mathrm{F}(H) \\
& B_{H_{1}, B_{1}}^{d}=B_{d}(H)-B_{d}\left(-H_{1}\right)+B_{1}-2 \mathrm{~F}\left(H_{1}\right) \mathrm{F}(-H)
\end{aligned}
$$

Where, $F(H)$ can be deduced by formulas (4), (5):

$$
\begin{aligned}
& F(H)=\int_{H}^{H_{\text {sat }}} \mu_{\alpha}(\alpha) d \alpha \\
& =\left\{\begin{array}{cc}
\left(B_{d}(H) / 2-B_{u}(H) / 2\right) / \sqrt{B_{d}(H)} H \geq 0 \\
\sqrt{B_{d}(-H)} & H<0
\end{array}\right.
\end{aligned}
$$

The alternating sequence of the magnetization's local extrema is stored in a descending order and updated in stack form. Formulas (3, 4, 5, 6, 7, 8 and 9) are used to calculate the flux density when the core is not saturated, and when it is saturated, magnetization trajectory will turn into single valued, so it is easy to calculate using curve fitting method.

In conclusion, according to the Preisach Theory, CT core model can be established accurately by measuring the descending branch of limiting hysteresis loop and positive saturation magnetization curve, when structure of CT's core is fixed and the outside temperature does not change obviously.

\subsection{Modeling of closed-core CT based on Preisach theory}

CT's simplified equivalent circuit is shown in Fig. 3. In the figure, $i_{1}$ and $i_{m}$ are the primary and exciting currents which are both converted to secondary side; also, $i_{2}$ is the secondary current, $R_{2}$ and $L_{2}$ are the total resistance and total inductance of secondary side, and $L_{m}$ is the equivalent excitation inductance. The circuit equations are listed in Eqs. (10) and (11):

$$
\begin{aligned}
& \left\{\begin{aligned}
i_{2} R_{2}+L_{2} \frac{d i_{2}}{d t} & =N_{2} A \frac{d B}{d t} \\
i_{2}+i_{m} & =i_{1}
\end{aligned}\right. \\
& N_{2} A \frac{d B}{d t}+L_{2} \frac{d i_{m}}{d t}+i_{m} R_{2}=i_{1} R_{2}+L_{2} \frac{d i_{1}}{d t}
\end{aligned}
$$

In Eqs. (10) and (11), $N_{2}$ is the number of secondary coil, $A$ is the cross area of iron core, $B$ is magnetic flux density in the core. Discrete Eq. (11) as follow:

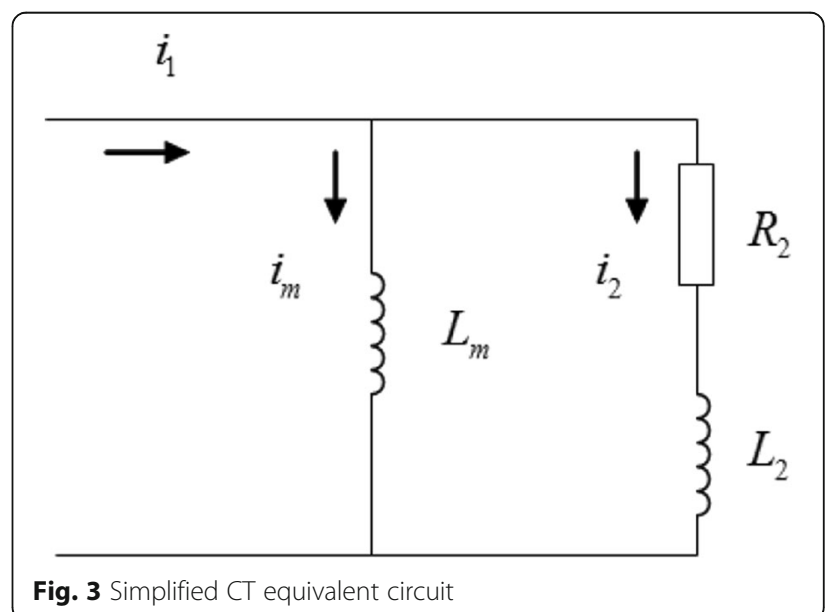




$$
\begin{aligned}
& N_{2} A F_{s}(B(k)-B(k-1))+L_{2} F_{s}+i_{m}(k) R_{2} \\
& =i_{1}(k) R_{2}+L_{2} F_{s}\left(i_{1}(k)-i_{1}(k-1)\right)
\end{aligned}
$$

$F_{s}$ is the sampling frequency. Equation (12) is called the solving equation of CT. Since the magnetic flux density $B$ increases monotonously with the exciting current, the solving equation can be solved in the following way: assume that the flux density and the exciting current at time instant $k$ are the same as at time instant $k-1$, which means substituting $i_{m}(k)=i_{m}(k-1)$ and $B(k)=B(k-1)$ to the left side of (12), then calculate the value of the right side, compare it with the left, if left < right, increase $i_{m}(k)$, otherwise decrease $i_{m}(k)$, till left $=$ right. The solving process is joint with core model, while the core model is air-gapped, air-gapped CT model is got, otherwise closed-core CT model is established. Core model based on Preisach Theory is established as follows.

Using the arctangent function and the hyperbolic function, the descending branch of limiting hysteresis loop of measured closed-core can be fitted. As shown in Fig. 4, the measured CT core is closed, with circular shape, the average magnetic path length $l=0.62 \mathrm{~m}$, sectional area $A=2.508 \times 10^{-3} \mathrm{~m}^{2}$.

The non-integer power is used to fit the measured positive saturation curve, as shown in Fig. 5. Using the fitting result, the values of flux density for arbitrary magnetization process can be calculated according to Preisach Theory. The displayed results in Figs. 6 and 7 show the magnetization curve and the waveform of applied magnetic field strength respectively.

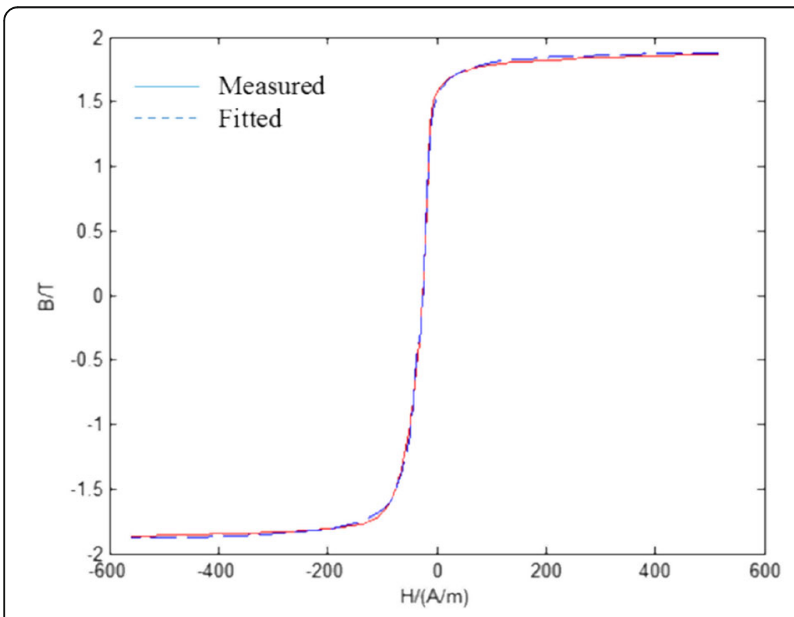

Fig. 4 Descending branch of limiting hysteresis loop of closed-core and its fitted curve

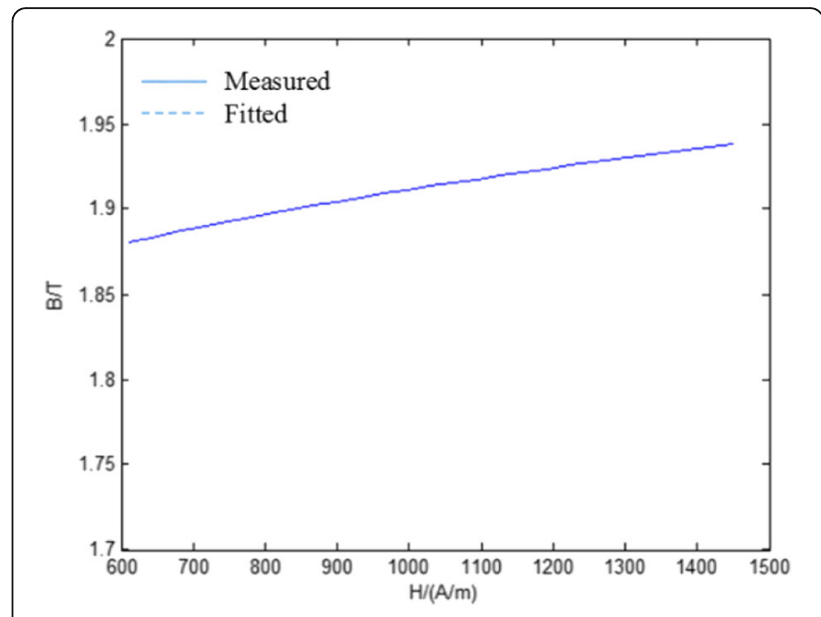

Fig. 5 Positive saturation curve of closed-core and its fitted curve

2.3 Modeling of air-gapped CT based on Preisach Theory The air-gapped CT core is shown in Fig. 8 , where $l_{0}$ is the gap length. Since the air gap length is much smaller than the total length of the magnetic circuit, typically about one thousandth of it, so the following assumptions can be made: the fracture surfaces are parallel and perpendicular to the magnetic field lines; the magnetic field lines at the edge of the gap will not protrude. The total length of magnetic circuit could be set as $l$, and length of magnetic circuit in core as $l_{1}$, magnetic field strength in core as $H_{1}$, and magnetic field strength in gap as $H_{0}$; hence, the exciting current converted to one turn is.

$$
i_{e}=H_{1} l_{1}+H_{0} l_{0}
$$

Where, $H_{0}=B / \mu_{0}$ and the magnetization curve of airgapped core could be calculated by:

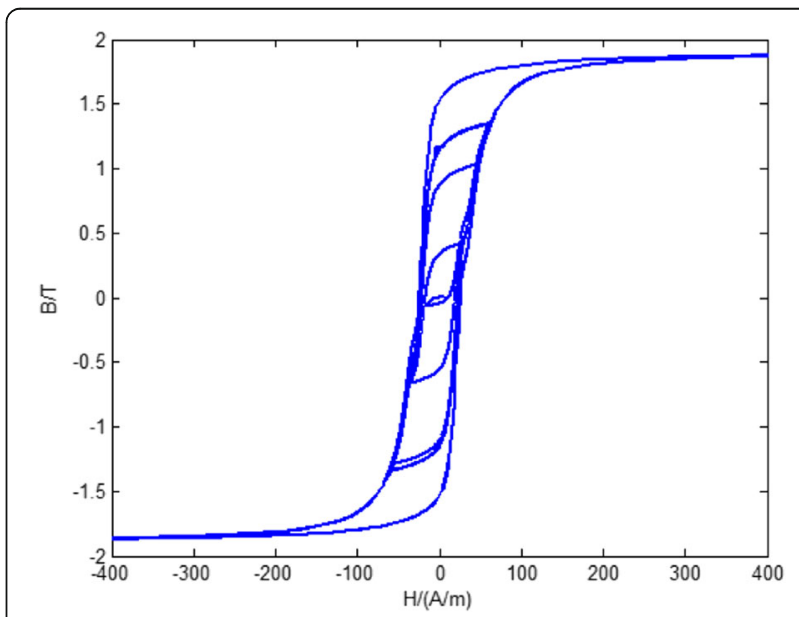

Fig. 6 Magnetization curve of closed-core CT model based on Preisach theory 


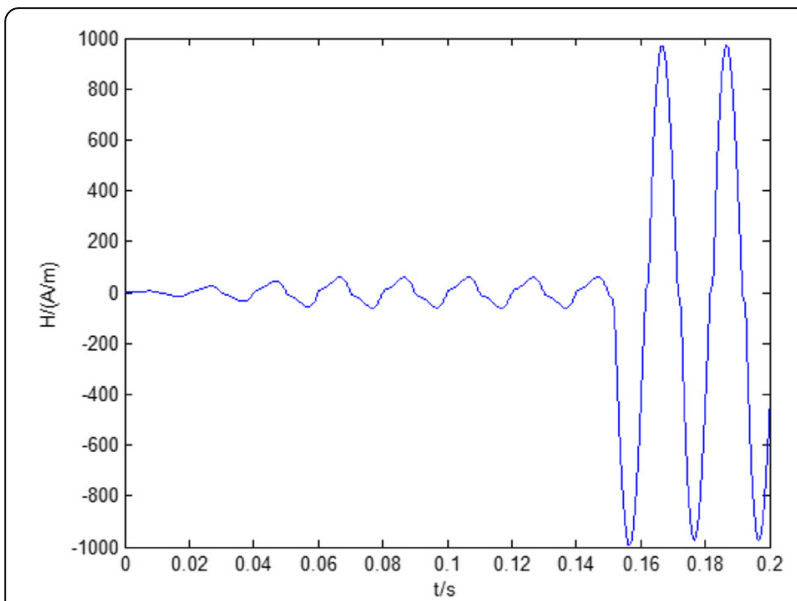

Fig. 7 Waveform of applied magnetic field strength on closed-core CT model

$$
H=F(B)=H_{1}+\frac{H_{0} l_{0}}{l}=f(B)+\frac{B l_{0}}{\mu_{0} l}
$$

In (14), $f(B)$ could be obtained from the inverse function of closed-core magnetization curve. In the case of the descending branch of closed-core's limiting hysteresis loop known, the descending branch of the limiting hysteresis loop of the air-gapped core with different lengths of air gap can be calculated by (14), as shown in Fig. 9.

In Fig. 9, waveforms a, b, and c are the descending branches of limiting hysteresis loop of the core with air gap ratios of $0.001,0.002$, and 0.003 , respectively. As can be seen in Fig. 9, the longer the air gap is, the greater the saturation magnetic field strength will be. In this paper, the iron core model is established by selecting the data of the core with the air gap ratio of 0.001 .

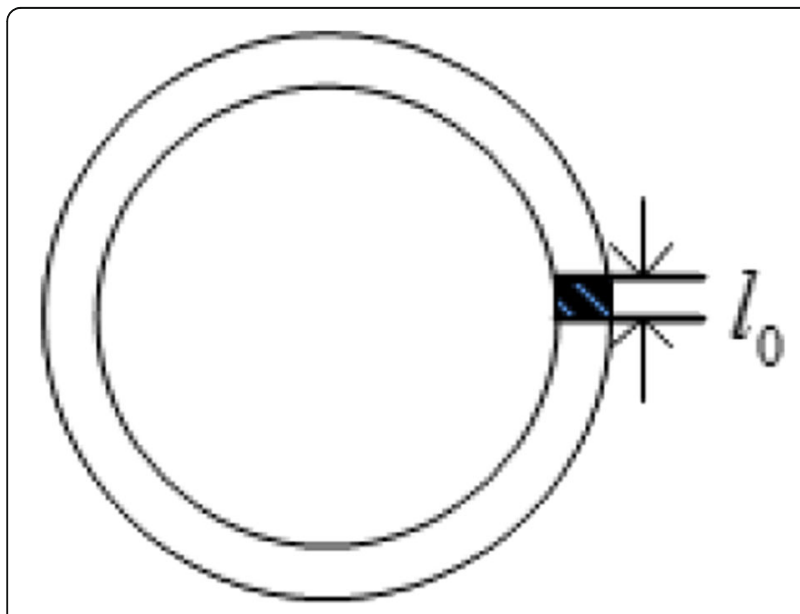

Fig. 8 Air-gapped core schematic diagram

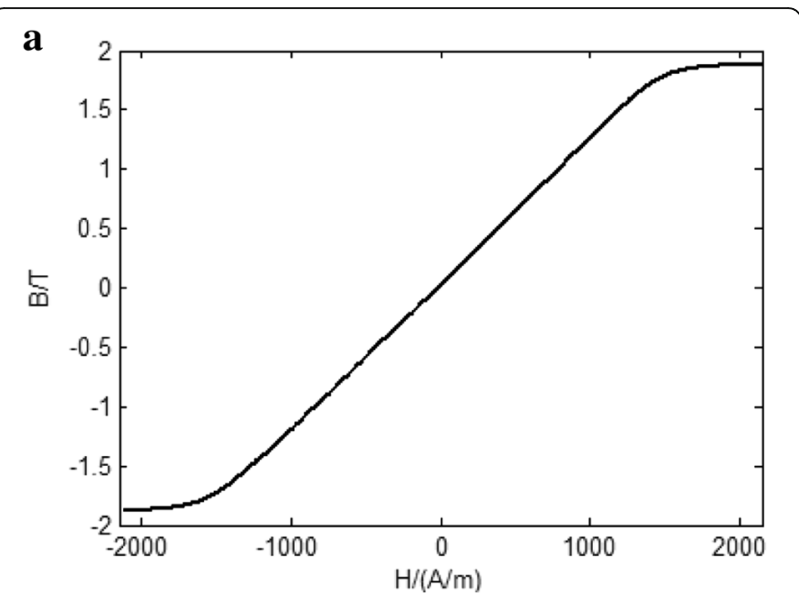

b
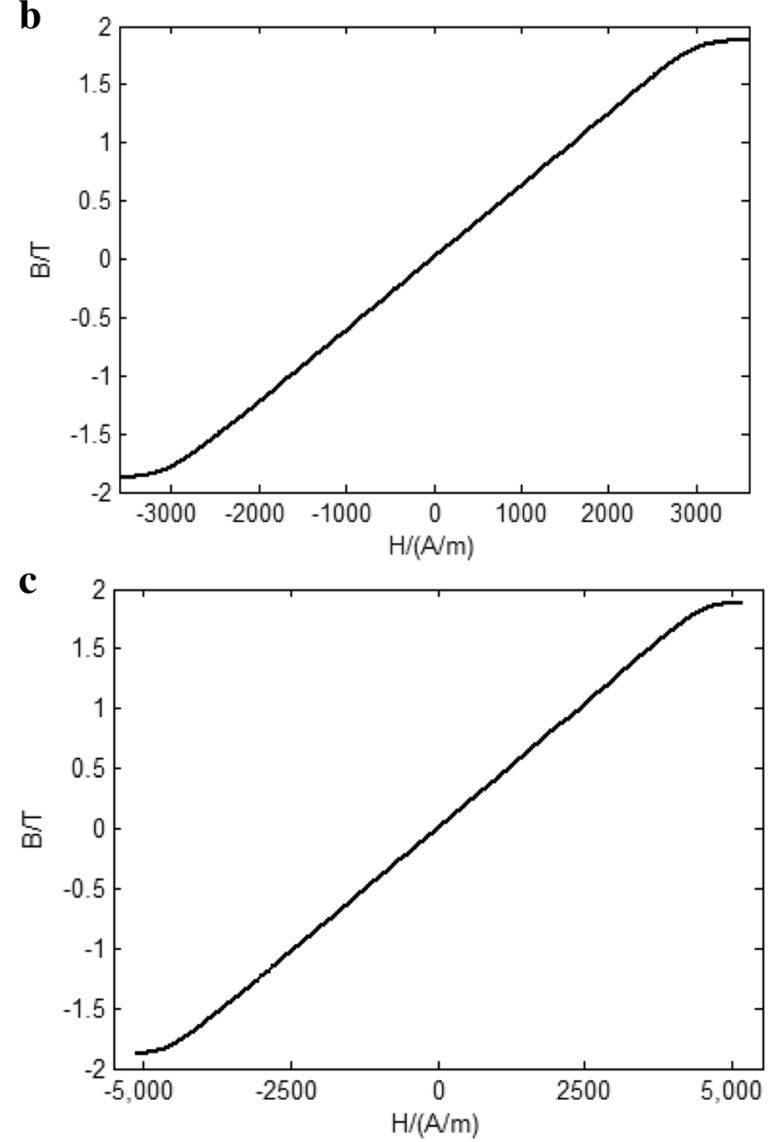

Fig. 9 The descending branch of limiting hysteresis loop of different air gap length ratios, a $0.001, \mathbf{b} 0.002, \mathbf{c} 0.003$

As a comparison, Fig. 10 shows the limiting hysteresis loops of closed core and air-gapped core with gap ratio of 0.0005 .

\section{Results}

\subsection{Characteristics of saturation}

A test circuit is simulated in PSCAD/EMTDC to generate different primary currents, as shown in Fig. 11. The closed-core CT model and the air-gapped core CT 


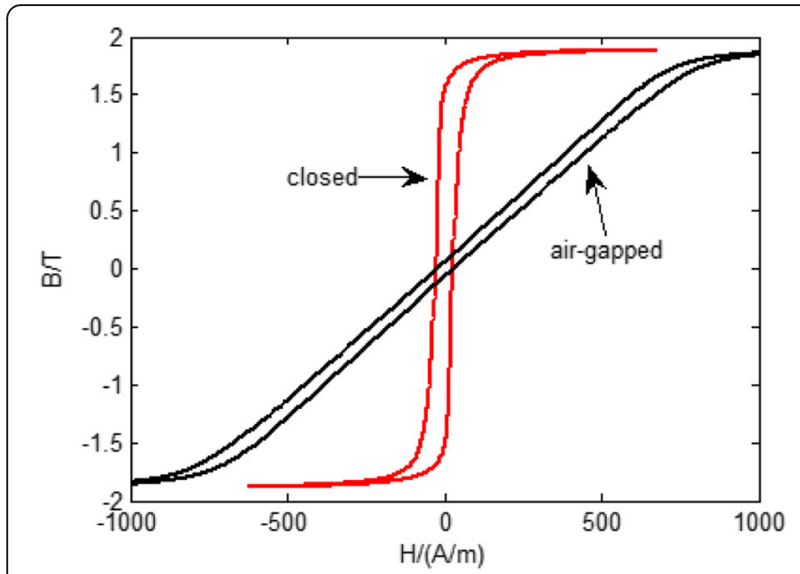

Fig. 10 Comparison of limiting hysteresis loops of closed core and air-gapped core with gap ratio of 0.0005

model are inputted respectively with data of primary current to compare the saturation characteristics and remanence characteristics. Parameters are set as: current ratio 1: 500 , load resistance $4 \Omega$, coil resistance $1 \Omega$, the load inductance and leakage inductance of the secondary side amount to $0.8 \mathrm{mH}$.

Characteristics of saturation are shown in Figs. 12 and 13. A-G fault occurs at $0.1 \mathrm{~s}$, and the fault current contains a significant DC component. The primary current is converted to the secondary side.

The short dashed line in Fig. 12 is the curve of flux density in Tesla which its axis is shown on the right side. The solid line and the long dashed line are, respectively, primary and secondary current respectively in Amperes which their axis are depicted on the left side. It can be seen that the closed-core CT is saturated. The course of saturation can be analyzed from Figs. 12 and 13. In the first cycle after the fault, since the current transformer core has no remanence, the magnetic flux density increases from zero, and does not reach the saturation threshold in the first cycle, so the transformer is not saturated. Starting from the second cycle, due to the role of remanence caused by the first cycle, flux density gradually reaches the saturation threshold and exceed, so the core gets saturated. When primary current crosses zero and increases in reverse, the flux density decreases gradually until saturation is removed. The coordinate of the

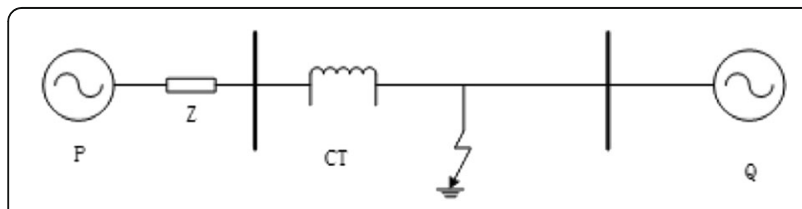

Fig. 11 Simulation circuit in PSCAD/EMTDC

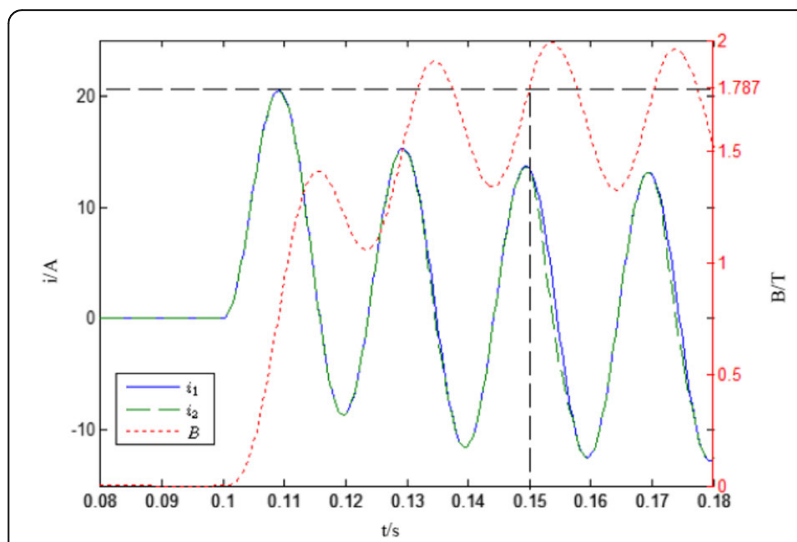

Fig. 12 Waveforms of closed-core CT's primary current, secondary current and magnetic flux density

saturation starting point $(0.15,1.787)$ for the third cycle is marked in Fig. 12.

\section{Discussion}

Since the core material and structure of both current transformer model are the same, so the critical saturation flux densities are also equal. As can be seen from Fig. 13, the core magnetic flux density does not reach a critical saturation value of $1.787 \mathrm{~T}$ at all time, and the magnetic flux density falls faster in the falling portion of each cycle than that of the closedcore $\mathrm{CT}$, thereby causing the rising portion to always fail in reaching the saturation threshold. The magnetic flux density in the air-gapped CT model reaches a maximum value of $1.657 \mathrm{~T}$ after the second cycle of the fault, and then the amplitude of the flux density gradually becomes smaller due to the decay of the DC component, and the transformer does not saturate. Even though the air-gapped CT shown in Fig. 13 suppresses the saturation due to

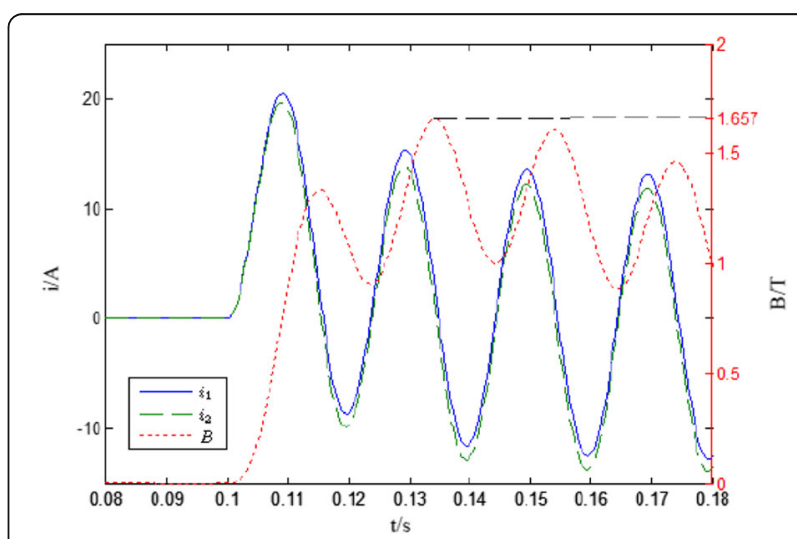

Fig. 13 Waveforms of air-gapped CT's primary current, secondary current and magnetic flux density 
the existence of the air gap, it also has the larger transmission error. This is because of the fact that the equivalent inductance of air-gapped core is smaller, resulting in fault current having a larger diversion in the excitation branch.

\subsection{Characteristics of remanence}

Remanence properties are divided into two aspects. The first one is the core remanence which affects on saturation during fault incident, and the second one is the elimination of the remanence during steady-state after fault clearance.

For the first aspect, the following four conditions have been simulated and compared: a) 50\% of the positive remanence, magnetic flux generated at fault occurring time is positive; b) $50 \%$ of the positive remanence, magnetic flux generated at fault occurring time is negative; c) $50 \%$ of the negative remanence, magnetic flux generated at fault occurring time is positive; d) $50 \%$ of the negative remanence, magnetic flux generated at fault occurring time is negative.

Figures 14, 15, 16 and 17 depict the results of the four groups of simulations; the first line in each group of results are magnetic flux density curve, and primary, secondary current comparison of closed-core CT, the second line in each group of results are magnetic flux density curve and primary and secondary current contrast of air-gapped CT.

As can be seen from Figs. 14, 15, 16 and 17, since the remanence level is low, $\pm 50 \%$ of the remanence is close to zero; thereforethe air-gapped CT does not saturate, regardless of the polarity of magnetic flux generated at fault occurring time same with remanence or not.

The closed-core CT is saturated when the remanence polarity is the same with the magnetic flux polarity of fault current at the moment of fault, and is saturated faster and deeper than in the case of zero remanence in Fig. 12, but is not saturated when the remanence polarity is opposite to the polarity of magnetic flux generated at fault occurring time. Comparing the two types of CT's flux density - time curves, it can be found that, in each case, the trend of the two are basically the same, but closed-core CT's flux density changes faster, can quickly reach the positive or negative saturation threshold, While the air-gapped core CT changes slower, with a smaller magnitude. This is because of the fact that the magnetization curve of the iron core with air gap becomes narrow and to reach the same magnetic flux density will require a larger excitation current. In other words, the iron core of the air-gapped CT is harder to be magnetized.

Then the characteristics of eliminating remanence after fault clearance are compared. The simulation conditions are as follows: the transmission line experience an A-G fault at $0.1 \mathrm{~s}$; the fault is cleared at $0.155 \mathrm{~s}$; total simulation time is $0.5 \mathrm{~s}$. The core remanence of both CTs are compared at the end of the simulation, and the results are shown in Fig. 18.

In Fig. 18, the dotted line and the solid line are changes of magnetic flux in the core of the closed-core
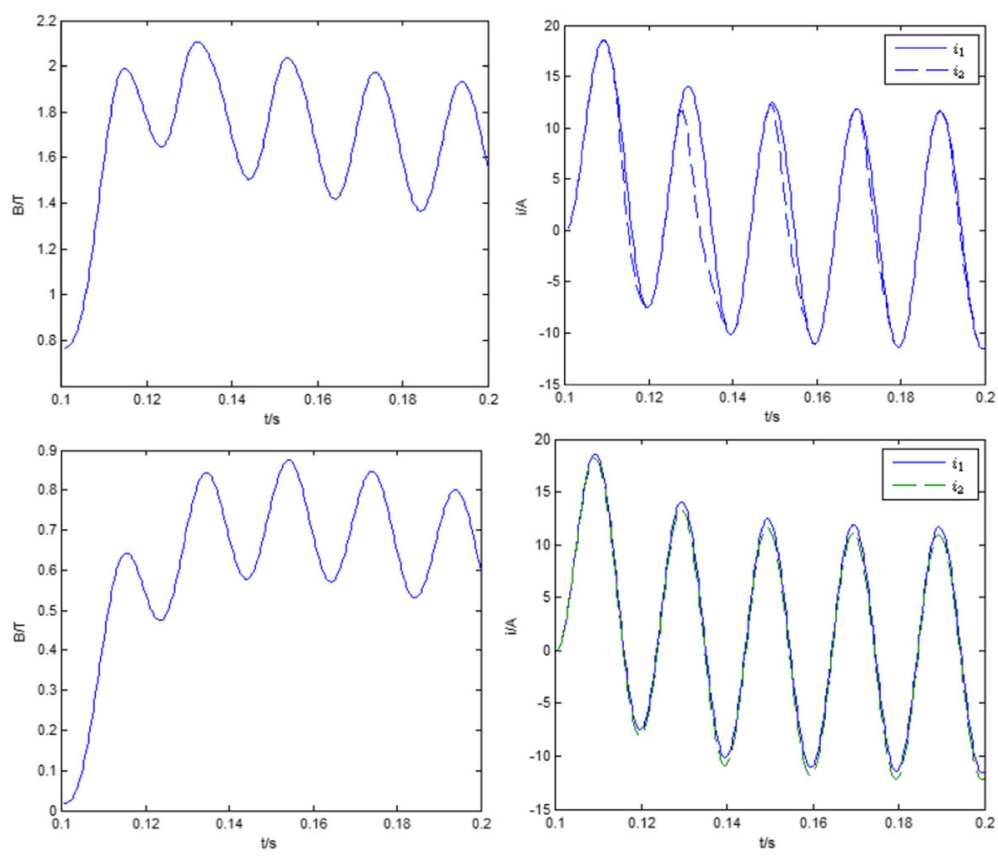

Fig. 14 50\% remanence, magnetic flux generated at fault occurring time is positive 

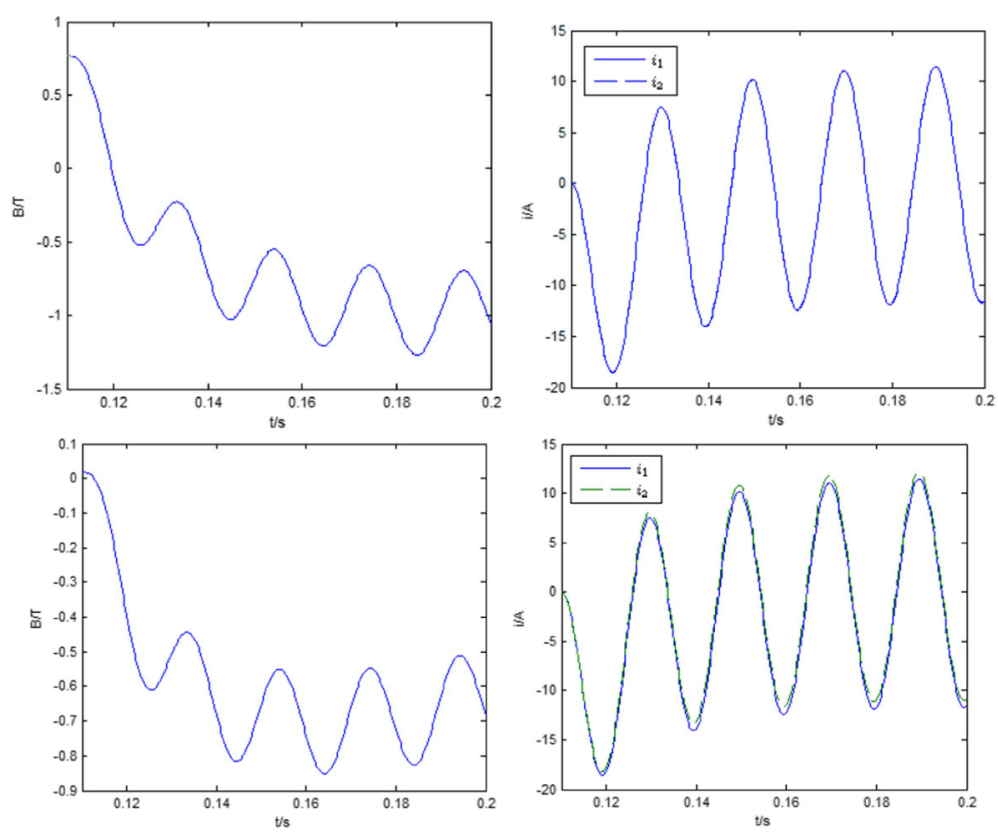

Fig. 15 50\% remanence, magnetic flux generated at fault occurring time is negative

$\mathrm{CT}$ and the air-gapped CT, respectively. According to Fig. 18, for air-gapped CT, the remanence of iron core decreases continuously with the increase of running time, and decreases to $0.127 \mathrm{~T}$ at $0.5 \mathrm{~s}$, which is about $7.1 \%$ of the saturation magnetic flux density; and for closed-core CT, the remanence is stable after the fault is removed, the minimum value of which is $1.312 \mathrm{~T}$, which is much larger than the air-gapped CT. When the subsequent fault occurs, if magnetic flux generated at fault occurring time is positive, it superimposes on the initial value of remanence, so the closed-core $\mathrm{CT}$ saturates easily. In the same condition, air-gapped CT core magnetic
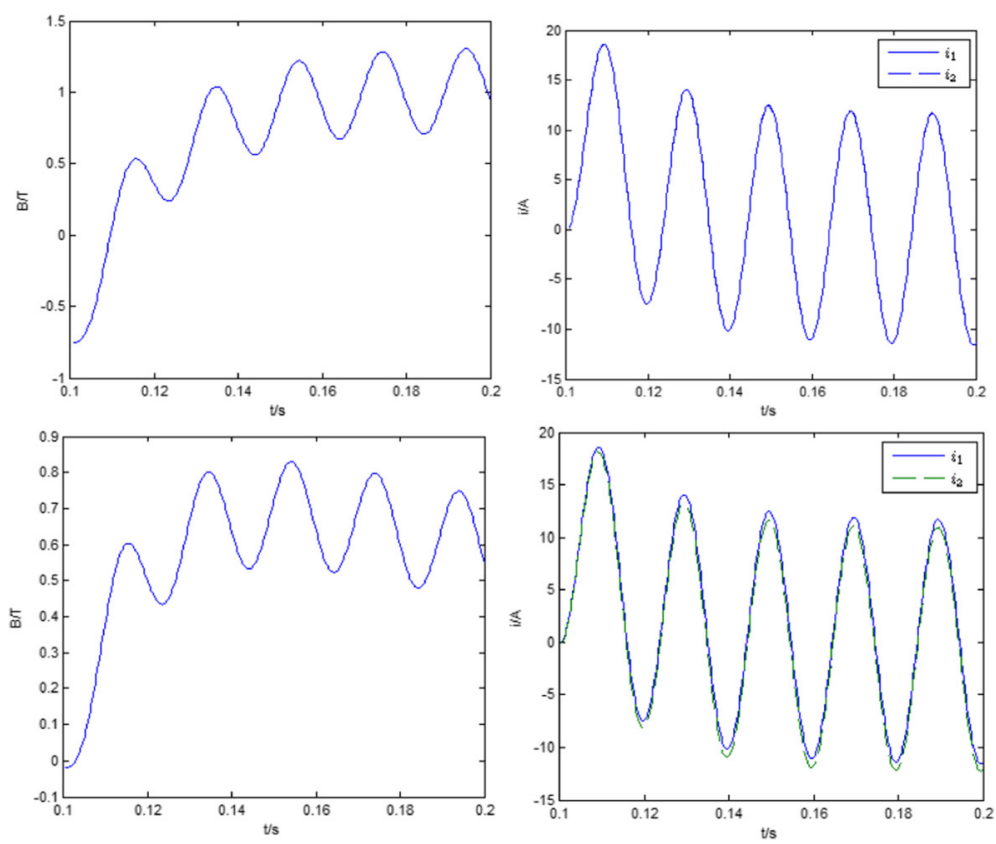

Fig. $16-50 \%$ remanence, magnetic flux generated at fault occurring time is positive 

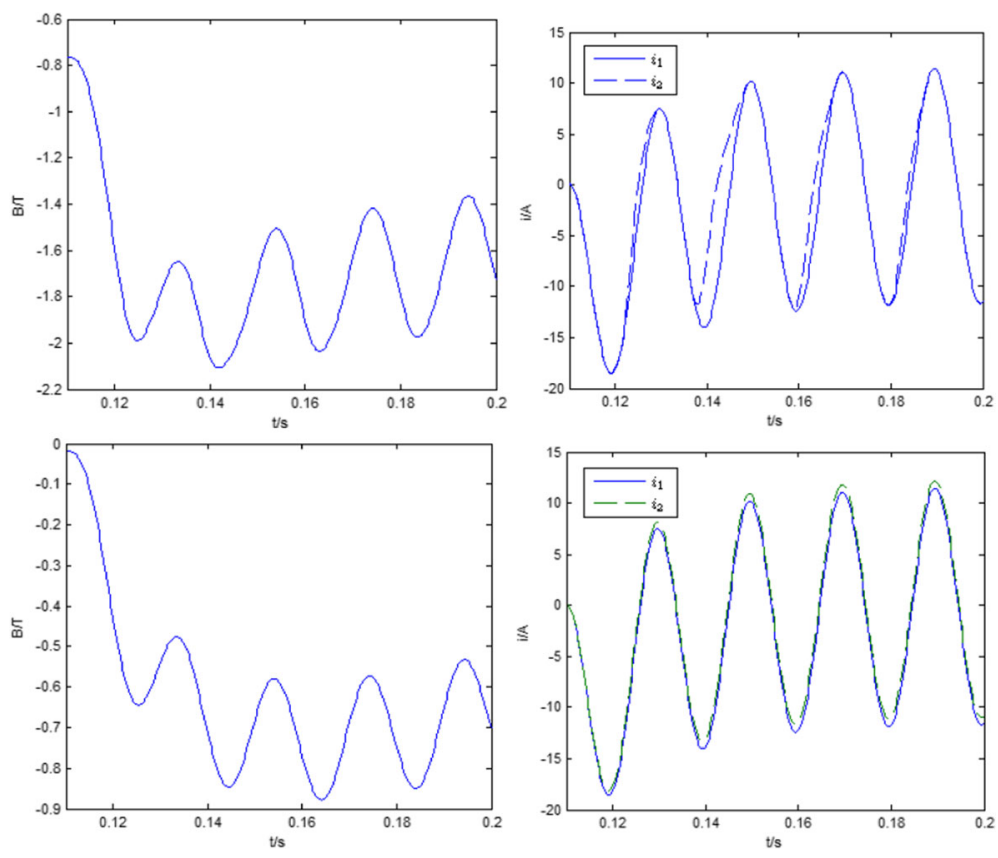

Fig. $17-50 \%$ remanence, magnetic flux generated at fault occurring time is negative

flux density will increase from the smaller value, $0.127 \mathrm{~T}$, more difficult to reach saturation. This phenomenon has been verified in the simulations below, as shown in Fig. 14.

Figures 19 and 20, respectively give the two types of CT's magnetization curve in the simulation. Figure 19a shows the magnetization curve of whole process of closed-core CT. It includes three areas, in which hysteresis loops are formed: oval box is for the loops before the fault, small hysteresis loops formed during steady-state operation; dashed rectangular box is for the loop of the first cycle after fault, and it could be seen that during the first cycle,

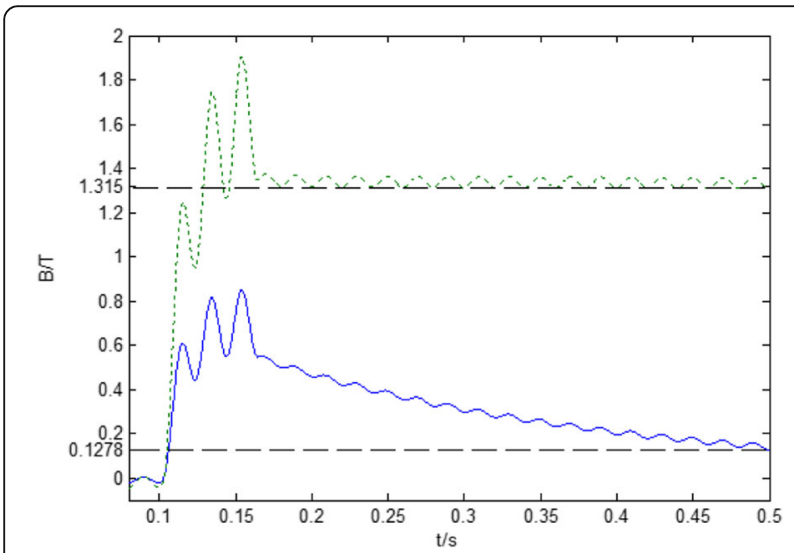

Fig. 18 Remanence comparison of iron cores in $\mathrm{CT}$ after fault clearance closed-core CT does not saturate, which is compatible with the result shown in Fig. 12; solid line box is for the loops after the fault clearance, which is enlarged to get Fig. 19b. It can be seen that the closed-core $\mathrm{CT}$, while entering into the steady sound state, runs near the remanence value in the form of small hysteresis loops. Considering both Figs. 18 and 19, shows that while closed-core CT transferring into the steady sound state, magnetic flux density in core is also falling with the increase of time, but the speed is very low, so there will be a lot of small hysteresis loops with small intervals.

Figure 20a shows the magnetization curve of whole process of air-gapped CT. The dotted box is the trajectory of the ending part of simulation and the solid box is the trajectory of the beginning part of simulation. Enlarging the ending part gets Fig. 20b. According to Fig. 20, the air-gapped CT's hysteresis loops are narrow, compared to the closed-core one. When the line fault is cleared, the core magnetic flux density continues to decline in an approximately spiral way, does not form a stable hysteresis loop. The core remanence value is gradually approaching zero. It can also be seen that the remanence of air-gapped CT can be gradually digested and, after the clearance it becomes lower than a certain level, usually $10 \%$ of saturation flux density.

\section{Conclusions}

Based on the Preisach core magnetization theory, the air-gapped core CT model is established, in which the 

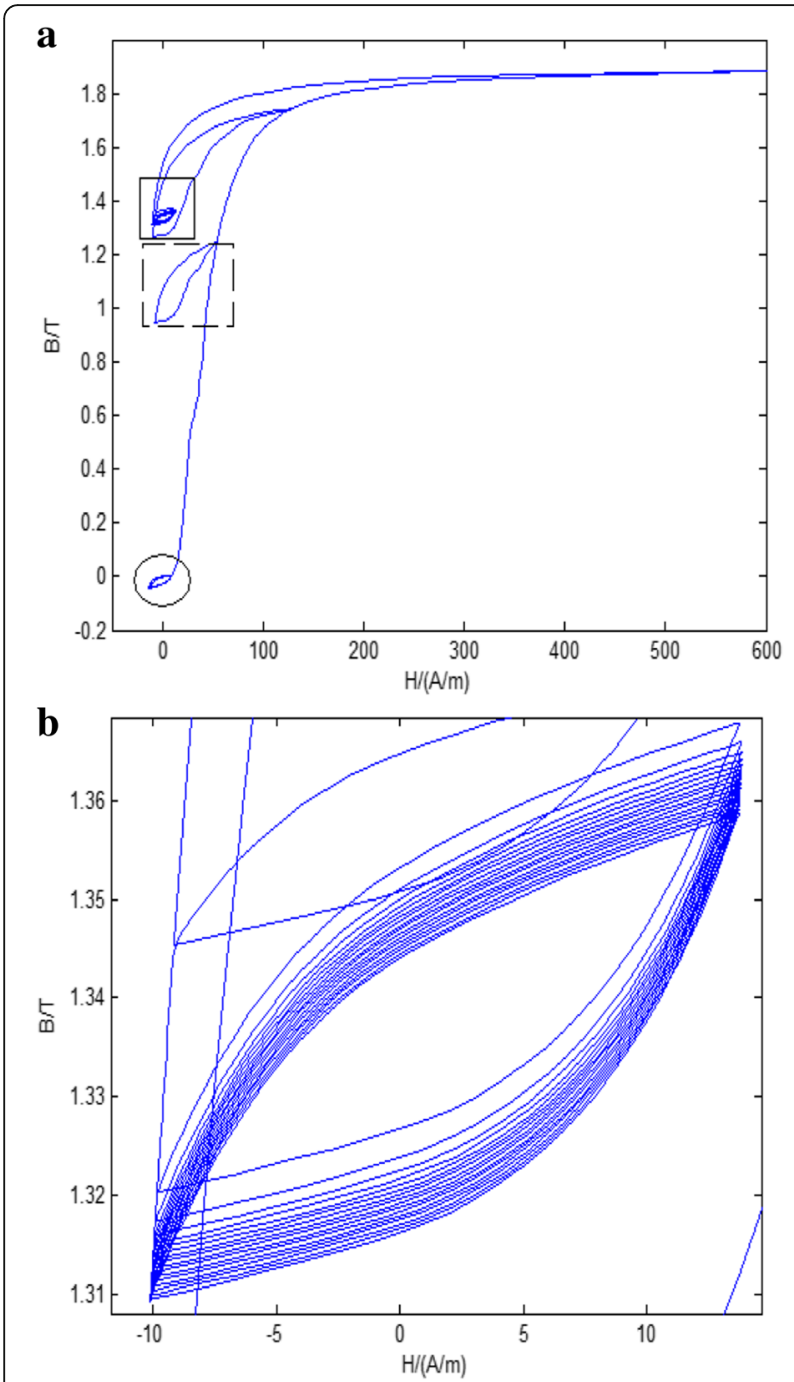

Fig. 19 Closed core magnetization curve. $\mathbf{a}$ whole process $\mathbf{b}$ ending part

data required for the core modeling is the descending branch of limiting hysteresis loop. The data can be obtained by experimental measurement, or from the closed-core CT with same core structure, shape and material. This model can correctly simulate the magnetization process of the air-gapped core, and the parameters can be set arbitrarily for simulation. The saturation characteristics and remanence characteristics of closed-core CT model and air-gapped CT model with same core structure, shape, material and parameters were simulated and compared. The results indicated the effects of air gap on CT performance, and proved the proposed model. The proposed modeling method can be utilized in CT selection and design, especially in CT design to analyze the necessary length of air gap in iron core. The correctness of the proposed method is based on the experimental validation of many existing papers

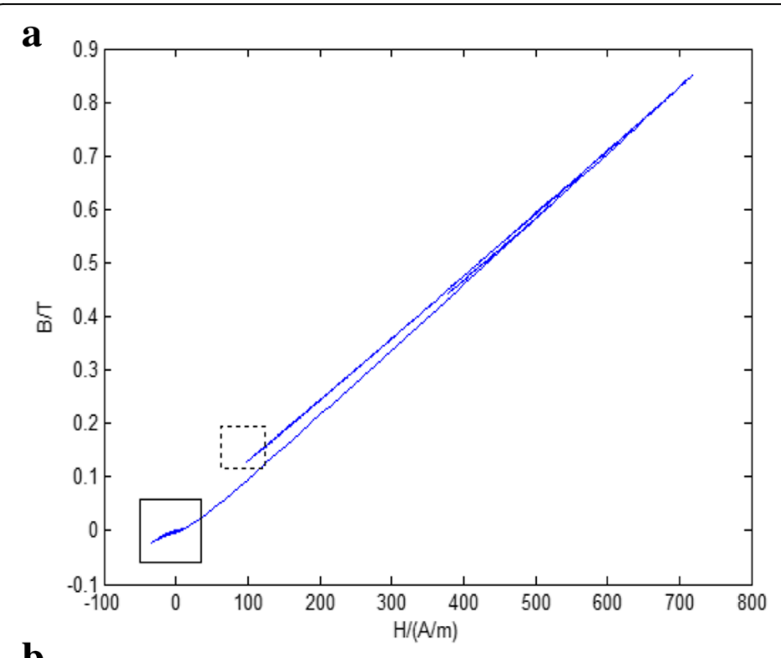

b

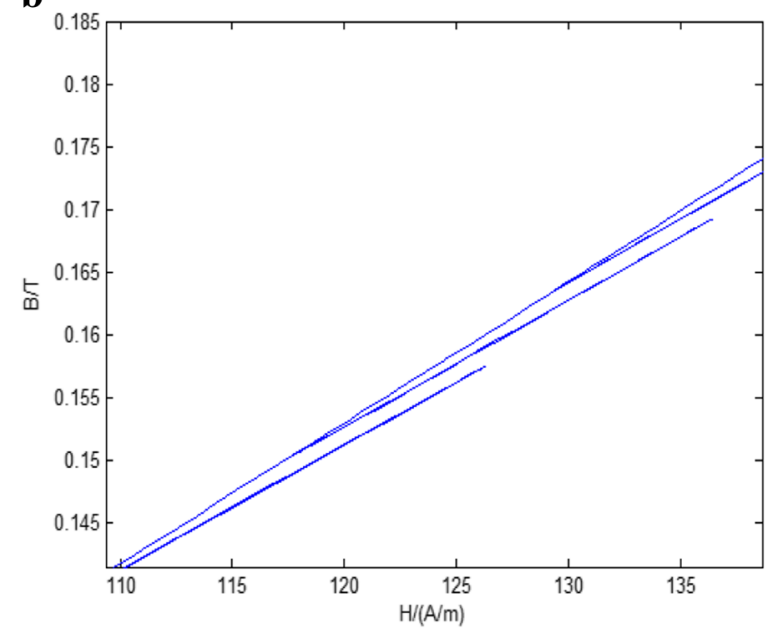

Fig. 20 Air-gapped core magnetization curve. $\mathbf{a}$ whole process $\mathbf{b}$ ending part

and the theoretical deducing and simulation of this paper, so the expectation of this method is, if possible, conducting an experiment with $\mathrm{CT}$ manufactures to measure the hysteresis trajectory of an iron core before and after opening an air gap in it. As a result, the proposed method can be validated in a further step.

\section{Funding}

This work was supported in part by the National Natural Science Foundation of China (Grant No. 51120175001), and in part by Science and Technology Project of State Grid Corporation of China (GWKJ2013-005).

\section{Authors' contributions}

Ya Hui Wu investigated the research status of air-gapped TA modeling, and established the proposed air-gapped TA model, then tested it with simulation and drafted the manuscript. Xin Zhou Dong and Sohrab Mirsaeidi participated in typesetting and revision of the manuscript. All authors read and approved the final manuscript.

\section{Authors' information}

Ya Hui Wu(1988-), male, M. Sc. candidate in the department of electrical engineering, Tsinghua University. His research interest is on TA modeling and reconstruction of saturated secondary current. 
Xin Zhou Dong(1963-), male, Phd. and Professor, Fellow of IEEE. Major in protective relaying, fault location, and application of wavelet transformer in power systems

Sohrab Mirsaeidi(1987), male, Phd. and post-doctor of department of electrical engineering, Tsinghua University. Major in smart grids, renewable energy.

\section{Competing interests}

The authors declare that they have no competing interests.

Received: 14 December 2016 Accepted: 20 March 2017

Published online: 04 April 2017

\section{References}

1. Tziouvaras, D. A., McLaren, P., Alexander, G., et al. (2000). Mathematical models for current, voltage, and coupling capacitor voltage transformers[J]. IEEE Transactions on Power Delivery, 15(1), 62-72.

2. Huang, L., Yang, W. X., \& Zhang, X. (2010). Modeling and simulation of current transformer with air-gap based on PSCAD/EMTDC[J]. Power system protection and control, 38(18), 178-182.

3. Muthumuni, D., McLaren, P. G., Chandrasena, W., et al. (2001). Simulation model of an air gapped current transformer[C]//Power Engineering Society Winter Meeting, 2001. IEEE. IEEE, 2, 705-709.

4. Yang, P. (2008). Research on physical mechanism and test method of heavy current transformer[D] (M. S. thesis). Beijing: Tsinghua University.

5. Zhang, L. (2013). Research on heavy current transformer's optimization and its simulation[D] (M. S. thesis). Beijing: Tsinghua University.

6. Mayergoyz, ID. (2003) Mathematical models of hysteresis and their applications[M]. New York: Academic Press.

7. Coulson, M. A., Slater, R. D., \& Simpson, R. R. S. (1977). Representation of magnetic characteristic, including hysteresis, using Preisach's theory. In Proceedings of the Institution of Electrical Engineers (Vol. 124(10), pp. 895-898). IET Digital Library.

8. Naidu, S. R. (1990). Simulation of the hysteresis phenomenon using Preisach's theory[J]. IEE Proceedings A-Physical Science, Measurement and Instrumentation, Management and Education, 137(2), 73-79.

9. Rezaei-Zare, A., Iravani, R., Sanaye-Pasand, M., et al. (2008). An accurate current transformer model based on Preisach theory for the analysis of electromagnetic transients[]]. IEEE Transactions on Power Delivery, 23(1), 233-242.

10. Matussek, R, Dzienis, C, Blumschein, J, et al. (2014). Current transformer model with hysteresis for improving the protection response in electrical transmission systems[C]//Journal of Physics: Conference Series. IOP Publishing, 570(6): 062001.

11. Eichler, J., Novák, M., \& Košek, M. (2016). Implementation of the first order reversal curve method for identification of weight function in Preisach model for ferromagnetics. In ELEKTRO (Vol. 2016, pp. 602-607).

12. Wiesen, K., \& Charap, S. H. (1988). A better scalar Preisach algorithm [J]. IEEE Transactions on Magnetics, 24(6), 2491-2493.

13. Cardelli, E., Torre, E. D., \& Tellini, B. (2000). Direct and inverse Preisach modeling of soft materials[J]. IEEE Transactions on Magnetics, 36(4), 1267-1271.

14. Bertotti, G. (1992). Dynamic generalization of the scalar Preisach model of hysteresis[J]. IEEE Transactions on Magnetics, 28(5), 2599-2601.

15. Cardelli, E., Fiorucci, L., \& Della, T. E. (2001). Identification of the Preisach probability functions for soft magnetic materials[J]. IEEE Transactions on Magnetics, 37(5), 3366-3369.

16. Zhang, X., Wang, Z., \& Xu, C. (2005). Preisach theory and its application to magnetization modeling of magnetic core[J]. High Voltage Engineering, 31(9), 14-17.

\section{Submit your manuscript to a SpringerOpen ${ }^{\circ}$ journal and benefit from:}

- Convenient online submission

- Rigorous peer review

- Immediate publication on acceptance

- Open access: articles freely available online

- High visibility within the field

- Retaining the copyright to your article 